Internat. J. Math. \& Math. Sci.

Vol. 23, No. 12 (2000) 801-806

S0161171200003781

(C) Hindawi Publishing Corp.

\title{
POSITIVE IMPLICATIVE ORDERED FILTERS OF IMPLICATIVE SEMIGROUPS
}

\author{
YOUNG BAE JUN and KYUNG HO KIM
}

(Received 10 August 1999)

\begin{abstract}
We introduce the notion of positive implicative ordered filters in implicative semigroups. We show that every positive implicative ordered filter is both an ordered filter and an implicative ordered filter. We give examples that an ordered filter (an implicative ordered filter) may not be a positive implicative ordered filter. We also give equivalent conditions of positive implicative ordered filters. Finally we establish the extension property for positive implicative ordered filters.
\end{abstract}

Keywords and phrases. Implicative semigroup, ordered filter, (positive) implicative ordered filter.

2000 Mathematics Subject Classification. Primary 20M12, 06F05, 06A06, 06A12.

1. Introduction. The notions of implicative semigroup and ordered filter were introduced by Chan and Shum [3]. The first is a generalization of implicative semilattice (see Nemitz [6] and Blyth [2]) and has a close relation with implication in mathematical logic and set theoretic difference (see Birkhoff [1] and Curry [4]). For the general development of implicative semilattice theory the ordered filters play an important role which is shown by Nemitz [7]. Motivated by this, Chan and Shum [3] established some elementary properties, and constructed quotient structure of implicative semigroups via ordered filters. Jun, Meng, and Xin [6] discussed ordered filters of implicative semigroups. Also Jun [6] stated implicative ordered filters of implicative semigroups. For a deep study of implicative semigroups it is undoubtedly necessary to establish more complete theory of ordered filters. In this paper, we introduce the notion of positive implicative ordered filters in implicative semigroups. We show that every positive implicative ordered filter is both an ordered filter and an implicative ordered filter. We give examples that an ordered filter (an implicative ordered filter) may not be a positive implicative ordered filter. We also give equivalent conditions of positive implicative ordered filters. Finally we establish the extension property for positive implicative ordered filters.

We recall some definitions and results.

By a negatively partially ordered semigroup (briefly, n.p.o. semigroup) we mean a set $S$ with a partial ordering " $\leq$ " and a binary operation "." such that for all $x, y, z \in S$, we have:

(1) $(x \cdot y) \cdot z=x \cdot(y \cdot z)$,

(2) $x \leq y$ implies $x \cdot z \leq y \cdot z$ and $z \cdot x \leq z \cdot y$,

(3) $x \cdot y \leq x$ and $x \cdot y \leq y$. 
An n.p.o. semigroup $(S ; \leq, \cdot)$ is said to be implicative if there is an additional binary operation $*: S \times S \rightarrow S$ such that for any elements $x, y, z$ of $S$,

(4) $z \leq x * y$ if and only if $z \cdot x \leq y$.

The operation $*$ is called implication. From now on, an implicative n.p.o. semigroup is simply called an implicative semigroup.

An implicative semigroup $(S ; \leq, \cdot, *)$ is said to be commutative if it satisfies

(5) $x \cdot y=y \cdot x$ for all $x, y \in S$, that is, $(S, \cdot)$ is a commutative semigroup.

In any implicative semigroup $(S ; \leq, \cdot, *)$, the following hold: for every $x, y \in S$,

(i) $x * x=y * y$,

(ii) $x * x$ is the greatest element, written 1 , of $(S, \leq)$.

Proposition 1.1 (see [3, Theorem 1.4]). Let $S$ be an implicative semigroup. Then for every $x, y, z \in S$, the following hold:

(6) $x \leq 1, x * x=1, x=1 * x$,

(7) $x \leq y *(x \cdot y)$,

(8) $x \leq x * x^{2}$,

(9) $x \leq y * x$,

(10) if $x \leq y$ then $x * z \geq y * z$ and $z * x \leq z * y$,

(11) $x \leq y$ if and only if $x * y=1$,

(12) $x *(y * z)=(x \cdot y) * z$,

(13) if $S$ is commutative then $x * y \leq(s \cdot x) *(s \cdot y)$ for all $s$ in $S$.

DEFINITION 1.2 (see [3, Definition 2.1]). Let $S$ be an implicative semigroup and let $F$ be a nonempty subset of $S$. Then $F$ is called an ordered filter of $S$ if

(F1) $x \cdot y \in F$ for every $x, y \in F$, that is, $F$ is a subsemigroup of $S$.

(F2) $x \in F$ and $x \leq y$, then $y \in F$.

The following result gives an equivalent condition of an ordered filter.

Proposition 1.3 (see [6, Proposition 2]). Suppose $S$ is an implicative semigroup. Then a nonempty subset $F$ of $S$ is an ordered filter if and only if it satisfies the following conditions:

(F3) $1 \in F$,

(F4) $x * y \in F$ and $x \in F$ imply $y \in F$.

DeFinITION 1.4 [5]. Let $S$ be an implicative semigroup. A nonempty subset $F$ of $S$ is called an implicative ordered filter of $S$ if it satisfies (F3) and

(I) $x *(y * z) \in F$ and $x * y \in F$ imply $x * z \in F$ for all $x, y, z \in S$.

Now we note important elementary properties of a commutative implicative semigroup, which follows from (5), (6), (10), and (12).

OBSERVATION 1.5. If $S$ is a commutative implicative semigroup, then for any $x, y$, $z \in S$,

(14) $x *(y * z)=y *(x * z)$.

(15) $x \leq(x * y) * y$.

(16) $y * z \leq(z * x) *(y * x)$.

(17) $y * z \leq(x * y) *(x * z)$.

(18) $((x * y) * y) * y=x * y$. 
TABLE 1.1.

\begin{tabular}{llllll}
\hline & l & a & b & c & d \\
\hline c & l & a & b & c & d \\
a & a & a & d & c & d \\
b & b & d & b & d & d \\
c & c & c & d & c & d \\
d & d & d & d & d & d \\
\hline
\end{tabular}

TABLE 1.2 .

\begin{tabular}{llllll}
\hline$*$ & 1 & $\mathrm{a}$ & $\mathrm{b}$ & $\mathrm{c}$ & $\mathrm{d}$ \\
\hline $\mathrm{l}$ & 1 & $\mathrm{a}$ & $\mathrm{b}$ & $\mathrm{c}$ & $\mathrm{d}$ \\
$\mathrm{a}$ & 1 & 1 & $\mathrm{~b}$ & $\mathrm{c}$ & $\mathrm{d}$ \\
$\mathrm{b}$ & 1 & $\mathrm{a}$ & 1 & $\mathrm{c}$ & $\mathrm{c}$ \\
$\mathrm{c}$ & 1 & 1 & $\mathrm{~b}$ & 1 & $\mathrm{~b}$ \\
$\mathrm{~d}$ & 1 & 1 & 1 & 1 & 1 \\
\hline
\end{tabular}

2. Positive implicative ordered filters. We start by defining a positive implicative ordered filter in an implicative semigroup.

MAIN DEFINITION. Let $S$ be an implicative semigroup. A nonempty subset $F$ of $S$ is called a positive implicative ordered filter of $S$ if it satisfies (F3) and

(Q) $x *((y * z) * y) \in F$ and $x \in F$ imply $y \in F$ for all $x, y, z \in S$.

EXAMPLE 2.1. Let $S:=\{1, a, b, c, d\}$ be a set with Cayley Tables 1.1 and 1.2 Hasse diagram (Figure 2.1) as follows:

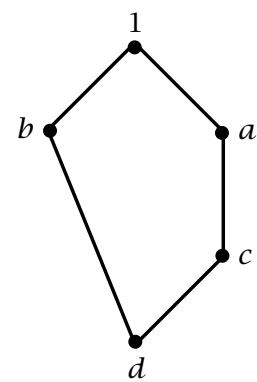

FIGURE 2.1.

It is easy to check that $S$ is a commutative implicative semigroup, and $F:=\{1, a, b\}$ is a positive implicative ordered filter of $S$.

THEOREM 2.2. Let $S$ be an implicative semigroup. Then every positive implicative ordered filter of $S$ is an ordered filter. 
Proof. Let $F$ be a positive implicative ordered filter of $S$ and let $x * y \in F$ and $x \in F$. Using (6) we have $x *((y * y) * y)=x * y \in F$ and $x \in F$. It follows from (Q) that $y \in F$, whence $F$ is an ordered filter of $S$.

LEMMA 2.3 (see [5, Theorem 1]). Let $S$ be an implicative semigroup and let $F$ be a nonempty subset of $S$. Then $F$ is an ordered filter if and only if it satisfies for all $x, y \in F$ and $z \in S$ :

(F5) $x \leq y * z$ implies $z \in F$.

THEOREM 2.4. Let $S$ be a commutative implicative semigroup. Then every positive implicative ordered filter of $S$ is an implicative ordered filter.

Proof. Let $F$ be a positive implicative ordered filter and let $x *(y * z) \in F$ and $x * y \in F$. Then

$$
\begin{aligned}
x *(y * z) & =y *(x * z) & & \text { [by (14)] } \\
& \leq(x * y) *(x *(x * z)) & & {[\text { by }(17)] . }
\end{aligned}
$$

Since $F$ is an ordered filter (see Theorem 2.2), it follows from Lemma 2.3 that $x *$ $(x * z) \in F$. On the other hand, note that

$$
\begin{aligned}
((x * z) * z) *(x * z) & =x *(((x * z) * z) * z) & & {[\text { by (14)] }} \\
& =x *(x * z) \in F & & {[\text { by (18)]. }}
\end{aligned}
$$

Hence $1 *(((x * z) * z) *(x * z)) \in F$. Since $F$ is a positive implicative ordered filter, we have $x * z \in F$ by (Q). This completes the proof.

REMARK 2.5. The converse of Theorems 2.2 and 2.4 may not be true as shown in the following example.

EXAMPLE 2.6. Let $S$ be a commutative implicative semigroup as in Example 2.1. We know that $G:=\{1, b\}$ is an implicative ordered filter, and hence an ordered filter. But it is not a positive implicative ordered filter, since $b *((a * d) * a) \in G$ and $b \in G$, but $a \notin G$.

Now we give equivalent conditions for every ordered filter (implicative ordered filter) to be a positive implicative ordered filter.

THEOREM 2.7. Let $S$ be an implicative semigroup and let $F$ be an ordered filter of $S$. Then $F$ is a positive implicative ordered filter if and only if, for all $x, y \in S$,

(F6) $(x * y) * y \in F$ implies $x \in F$.

Proof. Assume that $F$ is a positive implicative ordered filter and let $(x * y) * x \in F$ for all $x, y \in S$. Then, by (6), we have $1 *((x * y) * x) \in F$. Since $1 \in F$, it follows from (Q) that $x \in F$, and (F6) holds.

Conversely, suppose that $F$ satisfies (F6). Let $x *((y * z) * y) \in F$ and $x \in F$ for all $x, y, z \in S$. Then $(y * z) * y \in F$ by (F4), which implies $y \in F$ by (F6). Hence $F$ is a positive implicative ordered filter and proof is complete.

THEOREM 2.8. Let $S$ be a commutative implicative semigroup. If $F$ is a positive implicative ordered filter, then it satisfies 
(F7) $(x * y) * y \in F$ implies $(y * x) * x \in F$ for all $x, y \in S$.

Proof. Suppose $F$ is a positive implicative ordered filter and let $(x * y) * y \in F$ for all $x, y \in S$. Since $x \leq(y * x) * x$ by (9), it follows from (10) that

$$
((y * x) * x) * y \leq x * y
$$

Then

$$
\begin{aligned}
(x * y) * y & \leq(y * x) *((x * y) * x) & & {[\mathrm{by}(16)] } \\
& =(x * y) *((y * x) * x) & & {[\mathrm{by}(14)] } \\
& \leq(((y * x) * x) * y) *((y * x) * x) & & {[\mathrm{by}(2.3) \text { and }(10)] . }
\end{aligned}
$$

By (F2) and (6) we have $1 *((((y * x) * x) * y) *((y * x) * x)) \in F$, whence $(y *$ $x) * x \in F$ by $(\mathrm{Q})$. This completes the proof.

LEMMA 2.9 (see [5, Proposition 3]). Let $S$ be an implicative semigroup. If $F$ is an implicative ordered filter of $S$, then it satisfies for all $x, y \in S$ :

(F8) $x *(x * y) \in F$ implies $x * y \in F$.

THEOREM 2.10. Let $S$ be a commutative implicative semigroup and let $F$ be an implicative ordered filter of $S$ satisfying (F7). Then $F$ is a positive implicative ordered filter of $S$.

Proof. Suppose $F$ is an implicative ordered filter satisfying (F7) and let $(x * y) *$ $x \in F$ for all $x, y \in S$. It is sufficient to show that $x \in F$ by Theorem 2.7. Note from (16) that $(x * y) * x \leq(x * y) *((x * y) * y)$. Thus, by (F2), we have $(x * y) *((x * y) * y) \in$ $F$, whence $(x * y) * y \in F$ by Lemma 2.9. Since $F$ satisfies (F7), we have

$$
(y * x) * x \in F .
$$

On the other hand, by (6), (9), and (10) we get

$$
(x * y) * x \leq y * x=1 *(y * x),
$$

and hence $1 *(y * x) \in F$ by (F2). Since $1 \in F$, it follows from (F4) that $y * x \in F$. Thus $x \in F$ follows from (2.5) and (F4). This completes the proof.

COROLLARY 2.11. Let $S$ be a commutative implicative semigroup and let $F$ be an implicative ordered filter of $S$. Then $F$ is a positive implicative ordered filter if and only if it satisfies (F7).

The following lemmas will be needed in the sequel.

LEMMA 2.12 (see [5, Theorem 8]). Let $S$ be a commutative implicative semigroup and let $F$ and $G$ be ordered filters of $S$ such that $F \subseteq G$. If $F$ is an implicative ordered filter, then so is $G$.

LEMMA 2.13 (see [5, Theorem 7]). Let $S$ be a commutative implicative semigroup and let $F$ be a nonempty subset of $S$. Then $F$ is an implicative ordered filter if and only if $F$ is an ordered filter, and $F$ satisfies for all $x, y, z \in S$ 
(F9) $x *(y * z) \in F$ implies $(x * y) *(x * z) \in F$.

Finally, we give the following theorem.

THEOREM 2.14 (extension property for positive implicative ordered filters). Let $S$ be a commutative implicative semigroup. If $F$ is a positive implicative ordered filter of $S$, then every ordered filter $G$ containing $F$ is also a positive implicative ordered filter.

Proof. Since every positive implicative ordered filter is an implicative ordered filter, by Lemma 2.12 we know that $G$ is an implicative ordered filter. Thus it is sufficient to show that $G$ satisfies (F7) (see Theorem 2.10). Assume that $a:=(x * y) * y \in G$ for all $x, y \in S$. Since $a *((x * y) * y)=1 \in F$ and $F$ is an implicative ordered filter, it follows from (F9) and (14) that

$$
(x *(a * y)) *(a * y)=(a *(x * y)) *(a * y) \in F .
$$

Since $F$ is a positive implicative ordered filter, by (F7) we get

$$
((a * y) * x) * x \in F \subseteq G .
$$

On the other hand,

$$
\begin{array}{rlrl}
(x * y) * y & =a & \\
& \leq(a * y) * y & & {[\text { by }(15)]} \\
& \leq(((a * y) * x) * x) *((y * x) * x) & & {[\text { by }(16)] .}
\end{array}
$$

Using (F2) we obtain $(((a * y) * x) * x) *((y * x) * x) \in G$, and by using (F4) we conclude that $(y * x) * x \in G$. Hence $G$ satisfies (F7), and the proof is complete.

\section{REFERENCES}

[1] G. Birkhoff, Lattice Theory, American Mathematical Society, Providence, R.I., 1967. MR 37\#2638. Zbl 153.02501.

[2] T. S. Blyth, Pseudo-residuals in semigroups, J. London Math. Soc. 40 (1965), 441-454. MR 31\#1211. Zbl 136.26903.

[3] M. W. Chan and K. P. Shum, Homomorphisms of implicative semigroups, Semigroup Forum 46 (1993), no. 1, 7-15. MR 93g:20127. Zbl 776.06012.

[4] H. B. Curry, Foundations of Mathematical Logic, McGraw-Hill Book Co., Inc., New York, 1963. MR 26\#6036. Zbl 163.24209.

[5] Y. B. Jun, Implicative ordered filters of implicative semigroups, Commun. Korean Math. Soc. 14 (1999), no. 1, 47-55. MR 2000c:06030.

[6] Y. B. Jun, J. Meng, and X. L. Xin, On ordered filters of implicative semigroups, Semigroup Forum 54 (1997), no. 1, 75-82. MR 98a:06022. Zbl 862.06005.

[7] W. C. Nemitz, Implicative semi-lattices, Trans. Amer. Math. Soc. 117 (1965), 128-142. MR 31\#1212. Zbl 128.24804.

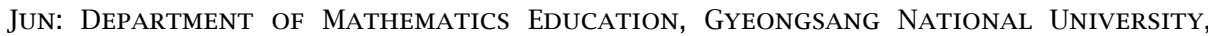
CHINJU 660-701, KOREA

E-mail address: ybjun@nongae.gsnu.ac.kr

Kim: Department of Mathematics, ChungJu National University, ChungJu 380-702, KOREA

E-mail address: ghkim@gukwon.chungju.ac.kr 


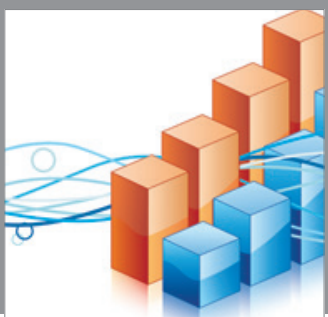

Advances in

Operations Research

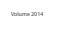

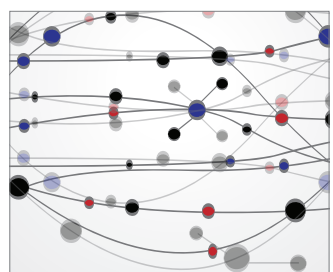

\section{The Scientific} World Journal
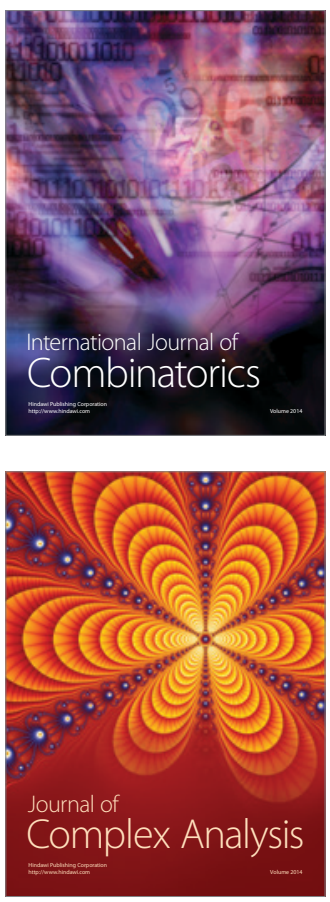

International Journal of

Mathematics and

Mathematical

Sciences
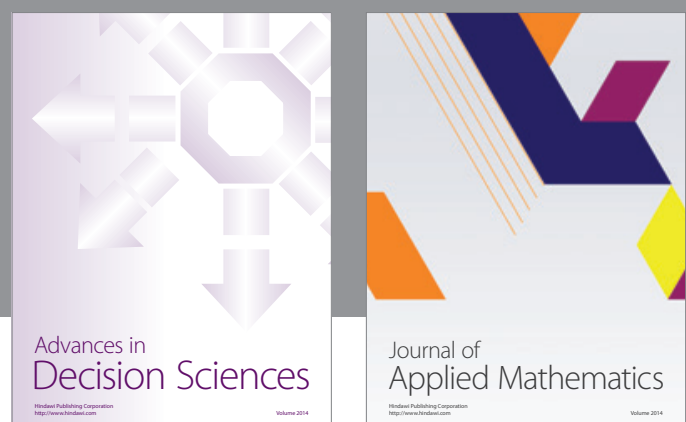

Journal of

Applied Mathematics
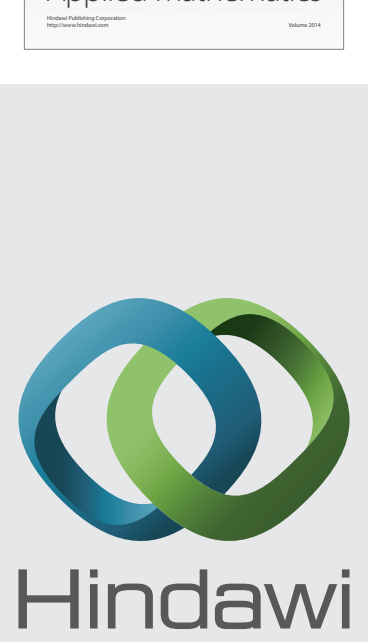

Submit your manuscripts at http://www.hindawi.com
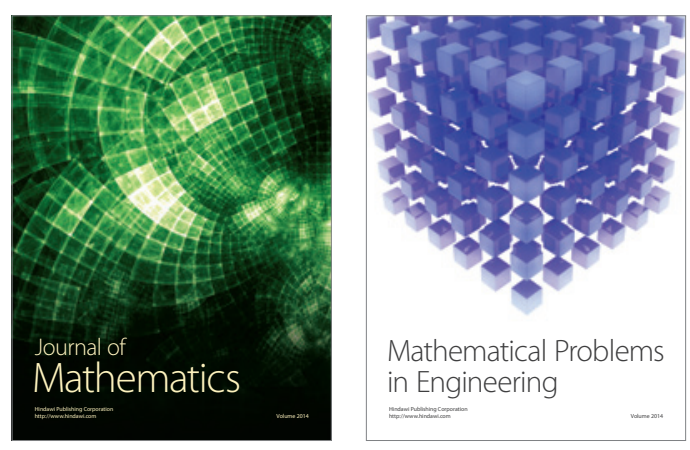

Mathematical Problems in Engineering
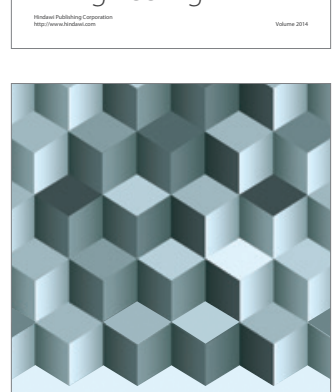

Journal of

Function Spaces
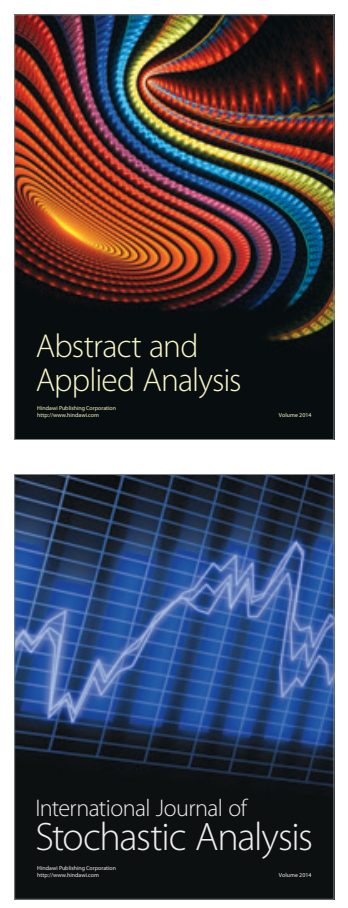

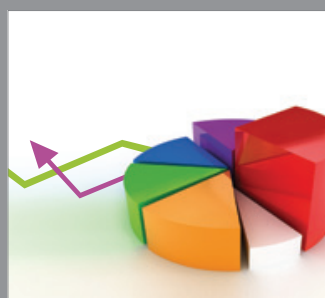

ournal of

Probability and Statistics

Promensencen
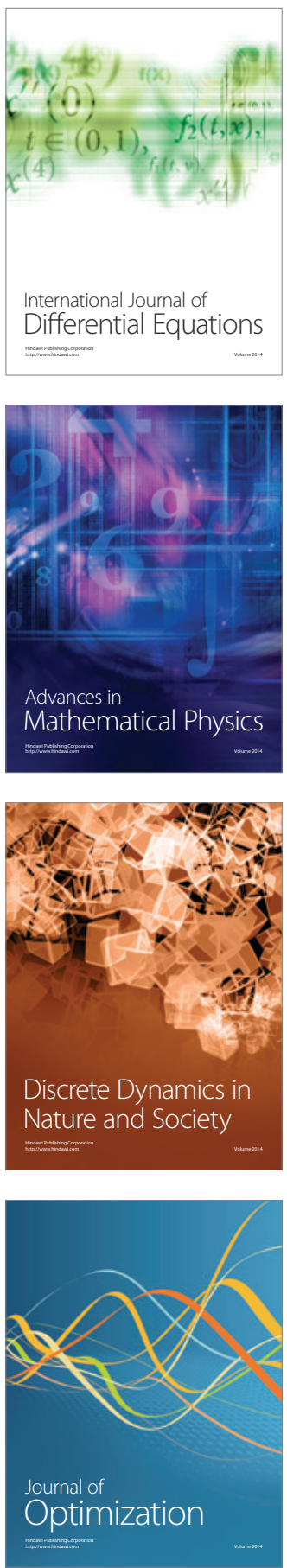